\title{
Usefulness of Ready-to-Use 0.4\% Sodium Hyaluronate (Endo-Ease) in the Endoscopic Resection of Gastrointestinal Neoplasms
}

\author{
Eun Ran Kim ${ }^{1, *}$, Yun Gyoung Park, ${ }^{1, *}$, Byung-Hoon Min ${ }^{1}$, Jun Haeng Lee ${ }^{1}$, Poong-Lyul Rhee ${ }^{1}$, Jae J. Kim ${ }^{1}$, Jung Ho Park², \\ Dong II Park ${ }^{2}$ and Dong Kyung Chang \\ ${ }^{1}$ Division of Gastroenterology, Department of Medicine, Samsung Medical Center, Sungkyunkwan University School of Medicine, Seoul, \\ ${ }^{2}$ Division of Gastroenterology, Department of Internal Medicine, Kangbuk Samsung Hospital, Sungkyunkwan University School of Medicine, \\ Seoul, Korea
}

Background/Aims: Commercially available sodium hyaluronate solutions are usually too thick to inject through catheters and need dilution with normal saline (NS) before use, which increases the risk of contamination. We evaluated the usefulness of ready-to-use $0.4 \%$ sodium hyaluronate, Endo-Ease (EE; UNIMED Pharm. Inc., Seoul, Korea).

Methods: We performed a prospective multicenter randomized study from May 2011 to September 2012. Patients requiring endoscopic resection (ER) for gastric or colorectal neoplasm at two referral hospitals were enrolled.

Results: One hundred fifty-four patients (72 with a gastric neoplasm and 82 with a colorectal neoplasm) were included in intentionto-treat analysis. Thirty-seven gastric neoplasms and 43 colorectal neoplasms were enrolled in the EE group. The usefulness rate was significantly higher in the EE group than in the NS group (89.2\% vs. $60.0 \%$ for gastric neoplasms and $95.3 \%$ vs. $67.7 \%$ for colorectal neoplasms, $p<0.001)$. In the EE group, the ease of mucosal resection was significantly higher than in the NS group ( $p<0.001)$. The injected volume was smaller in the EE group than in the NS group $(p<0.05)$.

Conclusions: The use of EE reduced the need for additional injections and improved the ease of ER. A submucosal injection of EE is useful for the ER of both gastric and colorectal neoplasms. Clin Endosc 2015;48:392-398

Key Words: Submucosal injection; Hyaluronic acid; Endoscopic resection

\section{INTRODUCTION}

Currently, endoscopic resection (ER) is the standard treatment for gastrointestinal neoplasms. En bloc resection of the lesion is important to provide accurate and reliable histopathological evaluation, and to achieve a successful treatment outcome. ${ }^{1}$ Hence, since the first description of endoscopic polypectomy as a treatment for pedunculated or semipedun-

Received: April 6, 2014 Revised: July 14, 2014

Accepted: July 24, 2014

Correspondence: Dong Kyung Chang

Division of Gastroenterology, Department of Medicine, Samsung Medical Center, Sungkyunkwan University School of Medicine, 81 Irwon-ro, Gangnam-gu, Seoul 06351, Korea

Tel: +82-2-3410-3409, Fax: +82-2-3410-6983, E-mail: dkchang@skku.edu

* Eun Ran Kim and Yun Gyoung Park contributed equally to this work as first authors.

(c) This is an Open Access article distributed under the terms of the Creative Commons Attribution Non-Commercial License (http://creativecommons.org/ licenses/by-nc/3.0) which permits unrestricted non-commercial use, distribution, and reproduction in any medium, provided the original work is properly cited. culated early gastric cancer was presented in 1974 in Japan, endoscopic techniques and devices have advanced to achieve en bloc resection regardless of lesion size and shape, permitting a curative resection. ${ }^{2}$ Recently, several types of electrosurgical knives, such as insulated-tipped diathermic knife, needle knife, hook knife, flex knife, or flush knife, have been developed and used.

However, the most important step prior to resection of a neoplasm is to separate the mucosal layer from the muscular layer. The submucosal injection of a solution to create a fluid cushion helps secure a clear-cutting view and helps reduce complications such as perforation, bleeding, and thermal injury. ${ }^{3,4}$ Numerous solutions have been developed and tested for use in ER. Normal saline (NS) solution is widely used; however, mucosal elevation is usually seen for only a short period after NS injection. ${ }^{5}$ Recently, several solutions, such as sodium hyaluronate, hydroxypropyl methylcellulose, mannitol, glycerol, and fibrinogen, have been investigated and 
shown to extend the duration of mucosal elevation. ${ }^{6}$ Sodium hyaluronate is considered the safest and most useful solution for submucosal injection during ER. However, it has several disadvantages: high cost, special requirements for preparation and storage, and lack of widespread availability. ${ }^{7.8}$ Endo-Ease is a newly developed ready-to-use sodium hyaluronate solution that is optimally diluted to $0.4 \%$ (UNIMED Pharm. Inc., Seoul, Korea), therefore overcoming the disadvantages of preparation and storage associated with conventional sodium hyaluronate.

In the current study, we aimed to identify the usefulness of Endo-Ease in the ER of both gastric and colorectal neoplasms as compared with NS in a multicenter prospective randomized controlled study.

\section{MATERIALS AND METHODS}

\section{Patients}

The target population of the study was consecutive patients who visited two referral hospitals (Samsung Medical Center and Kangbuk Samsung Hospital) in South Korea with gastric or colorectal neoplasms that could be treated endoscopically. Seventy-eight patients with a gastric neoplasm were enrolled from May 2011 to March 2012, and 86 patients with a colorectal neoplasm were enrolled from August 2011 to September 2012.

The inclusion criteria for a gastric neoplasm were differentiated intramucosal adenocarcinoma or adenoma of 5 to 20 $\mathrm{mm}$ diameter and patient age of 20 to 80 years. The inclusion criteria for a colorectal neoplasm were as follows: Paris type Isp, Is, IIa, IIb, or lateral spreading tumor lesion of $\leq 20 \mathrm{~mm}$ in diameter; lesion $<10 \mathrm{~mm}$ that includes a depressed area, such as Paris-type IIa+IIc or IIc+IIa; and patient age 20 to 80 years.

The following exclusion criteria were applied: (1) lesion accompanied by ulceration; (2) advanced malignant neoplasm; (3) significant comorbidity such as severe liver, kidney, or cardiovascular disease; (4) history of hypersensitivity to sodium hyaluronate; (5) bleeding tendency; (6) pregnant or lactating patient or woman who wished to become pregnant during the study; and (7) patients judged by a physician as inappropriate for inclusion.

ER procedures can be classified as either endoscopic mucosal resection (EMR) or endoscopic submucosal dissection (ESD). ESD consists of three steps: (1) injecting fluid into the submucosal layer to separate it from the proper muscle layer; (2) circumferential cutting of the mucosa surrounding the lesion; and (3) submucosal dissection of the connective tissue under the lesion with an electrosurgical knife. In the EMR procedure, a snare is used for resection instead of an electro- surgical knife. Circumferential cutting is performed in case of EMR with precutting. The choice of ER procedures was left to the discretion of the physicians at each facility.

The present study was conducted in compliance with the Helsinki Declaration and Good Clinical Practice. The institutional review boards of Samsung Medical Center and Kangbuk Samsung Hospital approved all study protocols. Written informed consent was obtained from all patients.

\section{Study device}

Endo-Ease is a manufactured product (UNIMED Pharm.) of $0.4 \%$ sodium hyaluronate containing sodium hyaluronate (80 mg), sodium chloride (170 mg), sodium hydrophosphate (11.2 mg), sodium dihydrophosphate ( $0.8 \mathrm{mg})$, and water $(18.67$ $\mathrm{mL})$. It is contained in a 10 or $20 \mathrm{~mL}$ glass vial, and stored at room temperature.

The upper limit of $0.4 \%$ sodium hyaluronate submucosal injection was $40 \mathrm{~mL}$, which is one-tenth the nontoxic level with intraperitoneal administration. An appropriate amount was used in each patient in the present study.

\section{Study design}

Randomization was performed by using telephone or fax at the central trial office. The patients were allocated by means of stratified block randomization.

The primary outcome measure was en bloc resection with a histopathologically negative resection margin, and the lifting and maintaining of a mucosal lesion during ER (evaluated according to the number of additional injections required owing to the loss of mucosal lesion lifting). The usefulness rate was defined as the percentage of en bloc complete resections that required an additional injection number of 0 or 1 during the procedure, and this rate was compared between the Endo-Ease and NS groups.

The secondary outcome measures included (1) ease of mucosal resection due to the use of the submucosal injection solution (excellent, good, moderate, or poor); (2) presence or absence of complications associated with the procedure, such as bleeding or perforation; (3) volume of submucosal injection solution used; and (4) time required for mucosal resection.

\section{Statistical analysis}

This study was designed with a level of significance of $\alpha=0.05$ and a detection power of $1-\beta=0.8$ to test the superiority of Endo-Ease compared with NS for lifting and maintaining the submucosa during ER. The calculated sample size was 78 patients for gastric neoplasms and 86 patients for colorectal neoplasms.

The data were analyzed with SAS software 9.1 version (SAS Institute, Cary, NC, USA). Clinical usefulness was assessed by 
using intention-to-treat analysis and as treated analysis, with the intention-to-treat analysis as the primary method. Continuous variables were compared with Student unpaired $t$-test or Wilcoxon rank sum test. The chi-square test or Fisher exact test was used for categorical variables. A $p<0.05$ was considered statistically significant.

\section{RESULTS}

\section{Patient characteristics}

A total of 162 patients (76 gastric neoplasms and 86 colorectal neoplasms) were enrolled in this study. Through randomization, 84 patients (39 gastric neoplasms and 45 colorectal neoplasms) were assigned to the Endo-Ease group and 78 patients (37 gastric neoplasms and 41 colorectal neoplasms) to the NS group. Among them, 154 patients (72 gastric neo-

\section{(A) Patients with a gastric neoplasm}

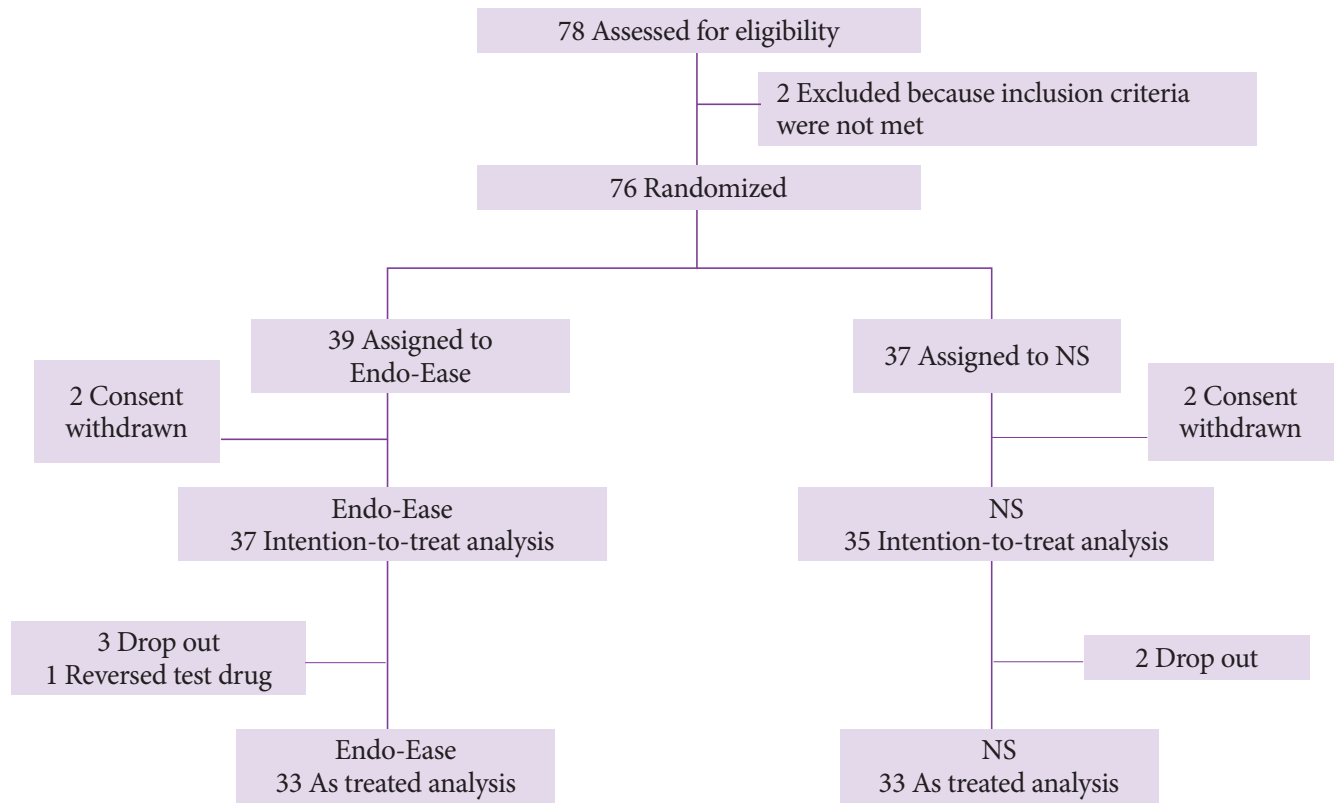

(B) Patients with a colorectal neoplasm

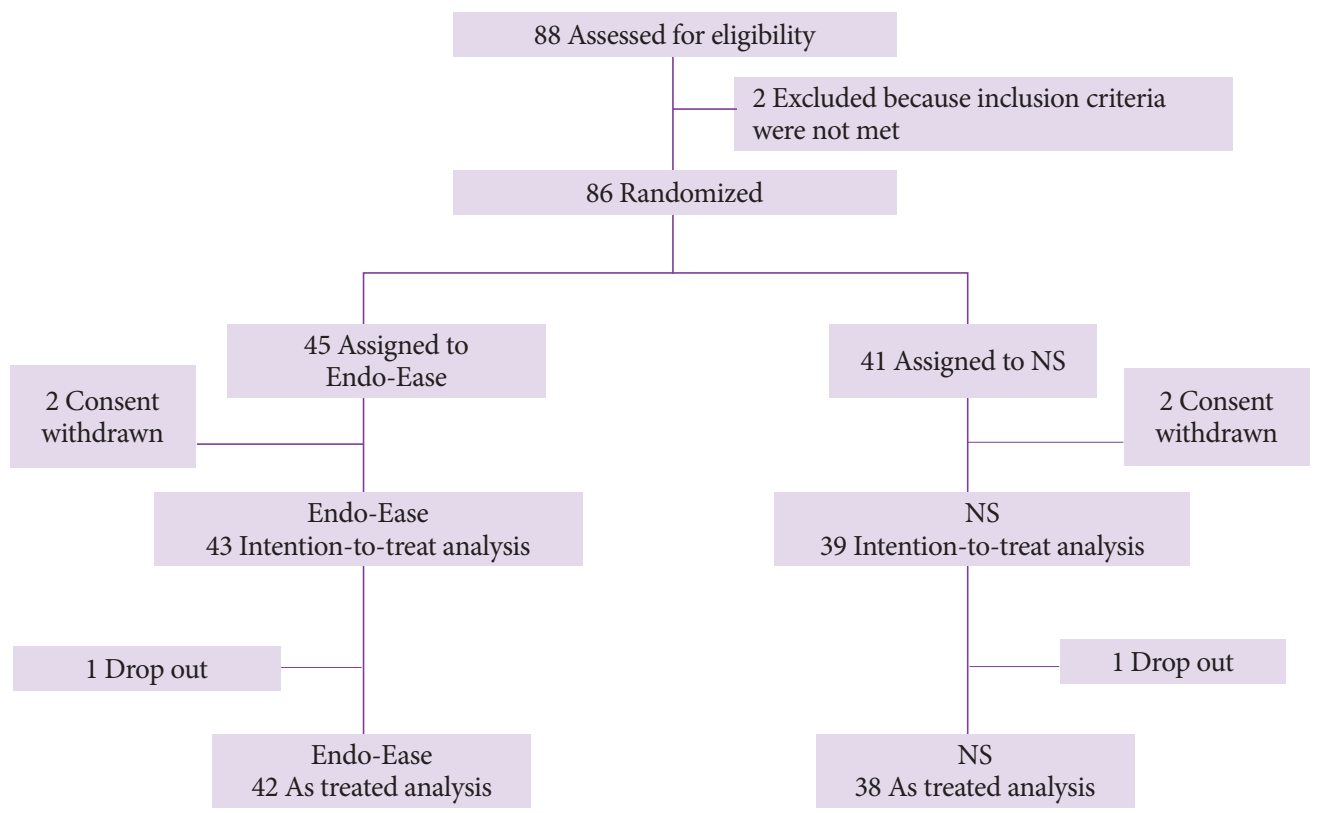

Fig. 1. Flow diagram of the randomization and progress of patients with a gastric neoplasm (A) and those with a colorectal neoplasm (B). NS, normal saline. 
Table 1. Clinical Characteristics of Patients with a Gastric Neoplasm

\begin{tabular}{lccc}
\hline Characteristic & $\begin{array}{c}\text { Endo-Ease } \\
(\boldsymbol{n}=\mathbf{3 9})\end{array}$ & $\begin{array}{c}\text { NS } \\
(\boldsymbol{n}=\mathbf{3 7})\end{array}$ & $\boldsymbol{p}$-value \\
\hline Age, mean \pm SD, yr & $63.77 \pm 9.66$ & $60.61 \pm 10.86$ & 0.12 \\
\hline Male sex, \% & 71.79 & 59.46 & 0.26 \\
\hline Location & & & 0.14 \\
\hline Proximal & 3 & 0 & \\
\hline Body & 7 & 10 & \\
\hline Distal & 29 & 27 & \\
\hline Macroscopic classification & & & 0.46 \\
\hline I & 0 & 2 & \\
\hline IIa & 14 & 11 & \\
\hline IIa+IIc/ IIc+IIa & 13 & 9 & \\
\hline IIb & 6 & 7 & \\
\hline IIc & 6 & 8 & \\
\hline Size, mm & & & 0.30 \\
\hline 5-10 & 11 & 17 & \\
\hline 11-15 & 16 & 11 & \\
\hline 16-20 & 12 & 9 & \\
\hline Histological type & & 19 & \\
\hline Adenoma & 20 & & \\
\hline Adenocarcinoma & 19 & & \\
\hline & & & \\
\hline
\end{tabular}

NS, normal saline.

plasms and 82 colorectal neoplasms) were included in the intention-to-treat analysis because eight patients withdrew consent (Fig. 1).

Table 1 shows the clinical characteristics of the Endo-Ease group and the NS group in patients with a gastric neoplasm. There were no statistically significant differences in the patient's age, sex, location, size of the lesion, or percentage of gastric cancer between the two groups.

The clinical characteristics of the patients with a colorectal neoplasm are shown in Table 2. In the Endo-Ease group, the ratio of male patients was higher than in the NS group $(76.6 \%$ vs. $56.4 \%$, respectively); however, there was no significant difference $(p=0.05)$. The location and size of the lesion, percentage of colorectal cancer, or macroscopic classification were not significantly different between the two groups.

\section{Clinical usefulness}

The primary outcome, usefulness, was assessed by using both intention-to-treat analysis and as treated analysis. The usefulness rate of the Endo-Ease group in patients with a gastric neoplasm was $89.2 \%$ (33 of 37) in the intention-to-treat analysis and $97.0 \%$ (32 of 33) in the as treated analysis. Both analyses showed that the usefulness rate of the Endo-Ease group was significantly greater than that of the NS group (21
Table 2. Clinical Characteristics of Patients with a Colorectal Neoplasm

\begin{tabular}{|c|c|c|c|}
\hline Characteristic & $\begin{array}{c}\text { Endo-Ease } \\
(n=45)\end{array}$ & $\underset{(n=41)}{N S}$ & $p$-value \\
\hline Age, yr & $58.95 \pm 10.66$ & $58.74 \pm 10.61$ & 0.93 \\
\hline Male sex, \% & 76.64 & 56.41 & 0.05 \\
\hline Location & & & 0.48 \\
\hline Cecum & $4(8.9)$ & $3(7.3)$ & \\
\hline A-colon & $9(20.0)$ & $9(22.0)$ & \\
\hline T-colon & $9(20.0)$ & $2(4.9)$ & \\
\hline D-colon & $1(2.2)$ & $2(4.9)$ & \\
\hline S-colon & $14(31.1)$ & $16(39.0)$ & \\
\hline Rectum & $8(17.8)$ & $9(22.0)$ & \\
\hline Macroscopic classification & & & 0.48 \\
\hline Ip & $4(8.9)$ & $3(7.3)$ & \\
\hline Isp & $9(20.0)$ & $9(22.0)$ & \\
\hline Is & $9(20.0)$ & $2(4.9)$ & \\
\hline IIa & $1(2.2)$ & $1(2.4)$ & \\
\hline Lateral spreading tumor & $14(31.1)$ & $16(39.0)$ & \\
\hline Other & $8(17.8)$ & $10(24.4)$ & \\
\hline Size, $\mathrm{mm}$ & & & 0.92 \\
\hline $5-10$ & $15(33.3)$ & $15(36.6)$ & \\
\hline $11-15$ & $21(46.7)$ & $19(46.3)$ & \\
\hline $16-20$ & $9(20.0)$ & 7 (17.1) & \\
\hline Histology & & & 0.22 \\
\hline Adenoma & $45(100)$ & $39(95.1)$ & \\
\hline Adenocarcinoma & 0 & $2(4.9)$ & \\
\hline
\end{tabular}

Values are presented as mean \pm SD or number (\%).

NS, normal saline; A-colon, ascending colon; T-colon, transverse colon; D-colon, descending colon; S-colon, sigmoid colon.

of $35,60 \%$ in the intention-to-treat analysis, $p=0.0027 ; 21$ of $33,63.6 \%$ in the as treated analysis, $p=0.0007$ ) (Table 3 ).

In patients with a colorectal neoplasm, the usefulness rate was $95.3 \%$ (41 of 43) in the Endo-Ease group and 67.7\% (26 of 39) in the NS group in the intention-to-treat analysis, and Endo-Ease was significantly more useful than NS ( $p=0.0002$ ). In the as treated analysis, the usefulness rate of the Endo-Ease group was significantly greater than that of the NS group (41 of $42,97.6 \%$ vs. 26 of $38,68.4 \%$, respectively; $p=0.0007$ ) (Table 4).

\section{Secondary outcomes}

The secondary outcomes analyzed included satisfaction with the ease of ER, volume of submucosal injection solution used, presence or absence of complications, and procedure time.

Satisfaction with the ease of submucosal injection in patients with a gastric neoplasm was significantly better in 
Table 3. Primary and Secondary Outcomes for Gastric Neoplasms

\begin{tabular}{|c|c|c|c|c|c|c|}
\hline \multirow[b]{2}{*}{ Variable } & \multicolumn{3}{|c|}{ Intention-to-treat analysis } & \multicolumn{3}{|c|}{ As treated analysis } \\
\hline & $\begin{array}{c}\text { Endo-Ease } \\
\quad(n=37)\end{array}$ & $\begin{array}{c}\text { NS } \\
(n=35)\end{array}$ & $p$-value & $\begin{array}{c}\text { Endo-Ease } \\
(n=33)\end{array}$ & $\underset{(n=33)}{\text { NS }}$ & $p$-value \\
\hline \multicolumn{7}{|l|}{ Primary outcome } \\
\hline Usefulness rate & $33(89.2)$ & $21(60.0)$ & $<0.01$ & $32(97.0)$ & $21(63.6)$ & $<0.01$ \\
\hline \multicolumn{7}{|l|}{ Secondary outcome } \\
\hline Ease of mucosal resection & & & $<0.01$ & & & $<0.01$ \\
\hline Excellent & $27(73.0)$ & $6(17.1)$ & & $26(78.8)$ & $6(18.2)$ & \\
\hline Good & $2(5.4)$ & $11(31.4)$ & & $1(3.0)$ & $9(27.3)$ & \\
\hline Moderate & $7(18.9)$ & $15(42.9)$ & & $6(18.2)$ & $15(45.5)$ & \\
\hline Poor & $1(2.7)$ & $3(8.6)$ & & 0 & $3(9.1)$ & \\
\hline Complication & $4(10.8)$ & $2(5.7)$ & 0.67 & $2(6.1)$ & $2(6.1)$ & 1.000 \\
\hline Injection volume, $\mathrm{mL}$ & & & $<0.05$ & & & $<0.05$ \\
\hline$<10$ & $4(10.8)$ & 0 & & $4(12.1)$ & 0 & \\
\hline $10-20$ & $9(24.3)$ & $4(11.4)$ & & $9(27.3)$ & $4(12.1)$ & \\
\hline $20-30$ & $10(27.0)$ & $9(25.7)$ & & $9(27.3)$ & $8(24.2)$ & \\
\hline$>30$ & $14(37.8)$ & $22(62.9)$ & & $11(33.3)$ & $21(63.6)$ & \\
\hline Procedure time, $\min$ & $34.19 \pm 21.94$ & $36.69 \pm 23.29$ & 0.64 & $31.85 \pm 19.21$ & $37.58 \pm 23.97$ & 0.34 \\
\hline
\end{tabular}

Values are presented as number (\%) or mean \pm SD.

NS, normal saline.

Table 4. Primary and Secondary Outcomes for Colorectal Neoplasms

\begin{tabular}{|c|c|c|c|c|c|c|}
\hline \multirow[b]{2}{*}{ Variable } & \multicolumn{3}{|c|}{ Intention-to-treat analysis } & \multicolumn{3}{|c|}{ As treated analysis } \\
\hline & $\begin{array}{c}\text { Endo-Ease } \\
(n=43)\end{array}$ & $\underset{(n=39)}{\mathrm{NS}}$ & $p$-value & $\begin{array}{l}\text { Endo-Ease } \\
\qquad(n=42)\end{array}$ & $\begin{array}{c}\text { NS } \\
(n=38)\end{array}$ & $p$-value \\
\hline \multicolumn{7}{|l|}{ Primary outcome } \\
\hline Usefulness rate & $41(95.3)$ & $26(67.7)$ & $<0.01$ & $41(97.6)$ & $26(68.4)$ & $<0.01$ \\
\hline \multicolumn{7}{|l|}{ Secondary outcome } \\
\hline Ease of mucosal resection & & & $<0.01$ & & & $<0.01$ \\
\hline Excellent & $38(88.4)$ & $14(35.9)$ & & $37(88.1)$ & $14(36.8)$ & \\
\hline Good & $4(9.3)$ & $16(41.0)$ & & $4(9.5)$ & $16(42.1)$ & \\
\hline Moderate & $1(2.3)$ & $7(18.0)$ & & $1(2.4)$ & $7(18.4)$ & \\
\hline Poor & 0 & $2(5.1)$ & & 0 & $1(2.6)$ & \\
\hline Complication & $4(9.3)$ & $3(7.7)$ & 1.00 & $4(9.52)$ & $2(5.3)$ & 0.68 \\
\hline Injection volume, $\mathrm{mL}$ & & & $<0.05$ & & & $<0.05$ \\
\hline$<10$ & $22(51.2)$ & $16(41.0)$ & & $21(50.0)$ & $16(42.1)$ & \\
\hline $10-20$ & $15(34.9)$ & $9(23.1)$ & & $15(35.7)$ & $9(23.7)$ & \\
\hline $20-30$ & $4(9.3)$ & $3(7.7)$ & & $4(9.5)$ & $3(7.9)$ & \\
\hline$>30$ & $2(4.7)$ & $11(28.2)$ & & $2(4.80)$ & $10(26.3)$ & \\
\hline Procedure time, min & $11.98 \pm 11.36$ & $9.79 \pm 6.53$ & 0.88 & $12.07 \pm 11.46$ & $9.56 \pm 6.41$ & 0.78 \\
\hline
\end{tabular}

Values are presented as number (\%) or mean \pm SD.

NS, normal saline.

the Endo-Ease group than in the NS group in both analyses $(p<0.001)$. In the intention-to-treat analysis of patients with a gastric neoplasm, an "excellent" grade was achieved in 73.0\%
(27 of 37) in the Endo-Ease group, whereas "excellent" was achieved only in $17.1 \%$ (6 of 35) in the NS group (Table 3). Similarly, in patients with a colorectal neoplasm, the satisfac- 
tion with the ease of submucosal injection was significantly higher in the Endo-Ease group than in the NS group ( $p<0.001)$. Satisfaction above the grade of "good" was achieved in $97.7 \%$ (42 of 43) in the Endo-Ease group and in 76.9\% (30 of 39) in the NS group in the intention-to-treat analysis (Table 4). The volume of submucosal injection solution was divided into four groups: $<10,10$ to 20,20 to 30 , and $>30 \mathrm{~mL}$. In patients with a gastric neoplasm, 37.8\% (14 of 37) in the Endo-Ease group needed $>30 \mathrm{~mL}$ of the submucosal injection solution, and $62.9 \%$ (22 of 35) of those in the NS group needed $>30 \mathrm{~mL}$ of the submucosal injection solution in the intention-to-treat analysis $(p=0.0416)$ (Table 3$)$. The results in patients with a colorectal neoplasm were similar to those with a gastric neoplasm. In the intention-to-treat analysis, $86.1 \%$ (37 of 43) of the Endo-Ease group with a colorectal neoplasm needed only $<20 \mathrm{~mL}$ of the submucosal injection solution, and $64.1 \%$ (25 of 39) of the NS group required $<20 \mathrm{~mL}$ of the submucosal injection solution $(p=0.0096)$.

Procedure-related complications occurred in 13 patients (10 bleedings and three perforations). All cases of bleeding developed during the procedure, and no delayed bleeding was observed. In patients with a gastric neoplasm, bleeding occurred in three patients (8.1\%) in the Endo-Ease group and in two patients (5.7\%) in the NS group. Perforation occurred in only one patient (2.7\%) in the Endo-Ease group. The rate of complications was not significantly different between the two groups in patients with a gastric neoplasm $(p=0.6745)$. In patients with a colorectal neoplasm, the rate of bleeding was not significantly different between the groups (3 of 43 [7\%] in the Endo-Ease group and 2 of 39 [5.1\%] in the NS group), and perforation occurred in one patient in each group. The rate of complications was not significantly different between the two groups in patients with a colorectal neoplasm $(p=1.000)$.

Procedure time was defined as the period from the marking of dots identifying the lesion in patients with a gastric neoplasm, or the beginning of submucosal injection in those with a colorectal neoplasm, to the completion of excision. In patients with a gastric neoplasm, the procedure time was $34.19 \pm 21.94$ minutes in the Endo-Ease group and 36.69 \pm 23.29 minutes in the NS group $(p=0.6440)$. In patients with a colorectal neoplasm, the procedure time was $11.98 \pm 11.36 \mathrm{~min}$ utes in the Endo-Ease group and 9.79 \pm 6.53 minutes in the NS group ( $p=0.8803$ ). There was no significant difference between the Endo-Ease and NS groups.

\section{DISCUSSION}

In this study, the usefulness of submucosal injection of Endo-Ease solution for the ER of gastric and colorectal neo- plasms was assessed in comparison with NS. The present study showed that Endo-Ease maintains an appropriate submucosal cushion for ER compared with NS, and the rates of complications and procedure time were acceptable.

The "ideal" solution for submucosal injection should have the following characteristics: it should (1) provide a thick submucosal fluid cushion for EMR; (2) remain in the submucosal space long enough to allow ER; and (3) preserve the tissue specimen and allow for precise pathological staging. ${ }^{4} \mathrm{NS}$ is commonly used to create a submucosal cushion because it is easily available, cheap, and causes minimal tissue injury owing to its isotonic property. ${ }^{4.5}$ However, it is rapidly absorbed by surrounding tissue. Therefore, the mucosal lift flattens rather quickly, and multiple injections are needed. ${ }^{5,10}$ Sodium hyaluronate is a macromolecular polysaccharide composed of D-glucuronate and $\mathrm{N}$-acetyl-D-glucosamine, which is found in human connective tissues and body fluids. ${ }^{3,11}$ It is not antigenic or toxic in humans, and does not cause tissue damage. ${ }^{8,12}$ Previous studies have demonstrated the usefulness of sodium hyaluronate as a submucosal injection solution. ${ }^{5,10-15}$ Yamamoto et al. ${ }^{16}$ showed that submucosal injection of $0.5 \%$ sodium hyaluronate is a reliable method, with a high en bloc resection rate (76\%) and complete resection rate (77\%) in gastric neoplasms. Hyun et al..$^{17}$ reported that mucosal elevation lasted longer with $0.1 \%$ sodium hyaluronate than with NS in their study with fresh mongrel transverse colon. However, a disadvantage of sodium hyaluronate is its high cost. Hence, researchers have tried to find the optimal concentration of the solution that can maintain the maximum lesion-lifting effect at the lowest cost. ${ }^{5,15}$ Recently, several studies have reported the efficacy of a solution of about $0.4 \%$ sodium hyaluronate. ${ }^{5,10,11,14}$ Kim et al. ${ }^{5}$ and Yamamoto et al..$^{14}$ stated that $0.4 \%$ sodium hyaluronate was effective in the formation and maintenance of sufficient mucosal lesion lifting for gastric intramucosal neoplasms. Hirasaki et al. ${ }^{11}$ and Kishihara et al. ${ }^{10}$ demonstrated that $0.4 \%$ sodium hyaluronate enabled sufficient lifting and reduced the need for additional injections for ER of colorectal intramucosal neoplasms.

Endo-Ease is a newly developed ready-to-use sodium hyaluronate that is optimally diluted to $0.4 \%$. Previously available sodium hyaluronate solutions are usually too thick to inject through catheters, and thus need dilution with NS before use. This additional dilution preparation is time and labor intensive, and increases the risk of contamination. In this study, we showed the usefulness of Endo-Ease as a submucosal injection solution for ER in both gastric and colorectal neoplasms. For the ER of gastric neoplasms, the usefulness rate of Endo-Ease, defined as the percentage of en bloc complete resection that required an additional injection number of 0 or 1 during the procedure, was $89.2 \%$, and this rate was comparable to the 
usefulness rate of $0.4 \%$ sodium hyaluronate reported in previous studies (88.4\% to $90.9 \%)$ and superior to that of NS.14 For the ER of colorectal neoplasms, the usefulness rate of Endo-Ease (95.3\%) was superior to the $82.5 \%$ reported by Hirasaki et al. ${ }^{11}$ and greater than that of NS (67.7\%). Satisfaction with the procedure was also higher with Endo-Ease than with NS for both the gastric and colorectal neoplasms. The rate of satisfaction above the grade of "good" in the current study $(78.4 \%$ and $97.7 \%$ in patients with gastric and colorectal neoplasm, respectively) was comparable or superior to that reported in previous studies. ${ }^{5,10,11,14}$ In addition, although complications occurred in 13 patients (10 bleedings and three perforations), the rate of complications was not significantly different between the Endo-Ease and NS groups in the current study.

Although these data point to the usefulness of Endo-Ease, some limitations need to be considered. Gastric neoplasms located in the distal third (antrum and angle) were technically easy to resect, and the submucosal cushion after submucosal injection relatively lasted longer than in lesions in the mid to upper third. In this study, the proportion of gastric lesions located in the distal third was relatively high (74.4\%). Hence, the satisfaction with Endo-Ease was relatively lower than that reported in a previous study ( $78.4 \%$ vs. $90.9 \%$, respectively), although it was higher than that with NS. If the proportion of gastric neoplasms located in the mid to upper third had been higher, the satisfaction with Endo-Ease and the usefulness rate would likely have been greater. The results in patients with a colorectal neoplasm were similar to those with a gastric neoplasm. When the lesions were smaller, the submucosal cushion was less important. Therefore, if this study had included larger lesions, such as lateral spreading tumors, the usefulness rate of Endo-Ease and the satisfaction with the procedure would likely have been better.

In conclusion, the use of Endo-Ease as a submucosal injection solution reduced the need for additional injections and improved the ease of mucosal resection. Submucosal injection of Endo-Ease will be useful for the ER of both gastric and colorectal neoplasms.

\section{Conflicts of Interest}

The authors were supported by grants from UNIMED Pharm. Inc.

\section{REFERENCES}

1. Min BH, Lee JH, Kim JJ, et al. Clinical outcomes of endoscopic submucosal dissection (ESD) for treating early gastric cancer: comparison with endoscopic mucosal resection after circumferential precutting (EMR-P). Dig Liver Dis 2009;41:201-209.

2. Kang KJ, Kim KM, Min BH, Lee JH, Kim JJ. Endoscopic submucosal dissection of early gastric cancer. Gut Liver 2011;5:418-426.

3. Uraoka T, Fujii T, Saito Y, et al. Effectiveness of glycerol as a submucosal injection for EMR. Gastrointest Endosc 2005;61:736-740.

4. Yeh RW, Triadafilopoulos G. Submucosal injection: safety cushion at what cost? Gastrointest Endosc 2005;62:943-945.

5. Kim YD, Lee J, Cho JY, et al. Efficacy and safety of 0.4 percent sodium hyaluronate for endoscopic submucosal dissection of gastric neoplasms. World J Gastroenterol 2013;19:3069-3076.

6. Al-Taie OH, Bauer Y, Dietrich CG, Fischbach W. Efficacy of submucosal injection of different solutions inclusive blood components on mucosa elevation for endoscopic resection. Clin Exp Gastroenterol 2012;5:43-48.

7. Fujishiro M, Yahagi N, Kashimura K, et al. Comparison of various submucosal injection solutions for maintaining mucosal elevation during endoscopic mucosal resection. Endoscopy 2004;36:579-583.

8. Lee SH, Park JH, Park do H, et al. Clinical efficacy of EMR with submucosal injection of a fibrinogen mixture: a prospective randomized trial. Gastrointest Endosc 2006;64:691-696.

9. The Paris endoscopic classification of superficial neoplastic lesions: esophagus, stomach, and colon: November 30 to December 1, 2002. Gastrointest Endosc 2003;58(6 Suppl):S3-S43.

10. Kishihara T, Chino A, Uragami N, et al. Usefulness of sodium hyaluronate solution in colorectal endoscopic mucosal resection. Dig Endosc 2012;24:348-352

11. Hirasaki S, Kozu T, Yamamoto H, et al. Usefulness and safety of $0.4 \%$ sodium hyaluronate solution as a submucosal fluid "cushion" for endoscopic resection of colorectal mucosal neoplasms: a prospective multi-center open-label trial. BMC Gastroenterol 2009;9:1.

12. Yamamoto H, Yube T, Isoda N, et al. A novel method of endoscopic mucosal resection using sodium hyaluronate. Gastrointest Endosc 1999;50:251-256.

13. Conio M, Rajan E, Sorbi D, et al. Comparative performance in the porcine esophagus of different solutions used for submucosal injection. Gastrointest Endosc 2002;56:513-516.

14. Yamamoto H, Yahagi N, Oyama $\mathrm{T}$, et al. Usefulness and safety of $0.4 \%$ sodium hyaluronate solution as a submucosal fluid "cushion" in endoscopic resection for gastric neoplasms: a prospective multicenter trial. Gastrointest Endosc 2008;67:830-839.

15. Fujishiro M, Yahagi N, Kashimura K, et al. Different mixtures of sodium hyaluronate and their ability to create submucosal fluid cushions for endoscopic mucosal resection. Endoscopy 2004;36:584-589.

16. Yamamoto H, Kawata H, Sunada K, et al. Success rate of curative endoscopic mucosal resection with circumferential mucosal incision assisted by submucosal injection of sodium hyaluronate. Gastrointest Endosc 2002;56:507-512.

17. Hyun JJ, Chun HR, Chun HJ, et al. Comparison of the characteristics of submucosal injection solutions used in endoscopic mucosal resection. Scand J Gastroenterol 2006;41:488-492. 